\title{
CÓMO ADQUIRIR COMPETENCIAS ESPECÍFICAS Y TRANSVERSALES A PARTIR DE LOS MASS MEDIA. UNA APLICACIÓN ORIGINAL DE APP EN LA UDG
}

Sílvia Espinosa-Mirabet ${ }^{1}$ : Universidad de Girona. España silvia.espinosam@udg.edu

Jaume Puig-Bargués: Universidad de Girona. España jaume.puig@udg.edu

Inés Ferrer-Real: Universidad de Girona. España ines.iferrer@udg.edu

Marianna Soler- i- Ortega: Universidad de Girona. España marianna.soler@udg.edu

Lluïsa Escoda: Universidad de Girona. España lluisa.escoda@udg.edu

Carmen Echazarreta-Soler: Universidad de Girona. España carmen.echazarreta@udg.edu

Rafael García-Campos Universidad de Girona. España rafael.garcia@udg.edu

\footnotetext{
${ }^{1}$ Autor correspondiente

Sílvia Espinosa-Mirabet: Departamento de Filología y Comunicación., Universidad de Girona, Girona (España).

Correo: silvia.espinosam@udg.edu
} 


\title{
RESUMEN
}

En este artículo se presenta un proyecto mutidisciplinar de APP que engloba disciplinas tan distintas como son Informática, Ingeniería, Física y Publicidad y que permite adquirir competencias específicas, propias de cada asignatura, y desarrollar competencias transversales comunes como: trabajar en equipo, diseñar propuestas creativas o comunicarse oralmente y por escrito. El proyecto tiene la originalidad de mezclar en un mismo trabajo a alumnos de segmentos universitarios diversos que de otra forma difícilmente trabajarían conectados, fomentando así la creatividad en un entorno científico. El objetivo del proyecto es transformar un programa de televisión de Karlos Arguiñano en un programa de radio. Para hacerlo los alumnos deben identificar, analizar y transformar los distintos aspectos científicos, técnicos y de comunicación que se observan en el programa televisivo para poderlo convertir en radiofónico. La elección de este eje vertebrador de APP es fruto de una larga reflexión para encontrar un proyecto real que se pudiese llevar a la práctica, aglutinando aspectos de las seis asignaturas de los diferentes ámbitos de conocimiento implicados en esta red de innovación docente. La implementación del APP, cada vez más usada en la universidad, es un proceso en el que se presenta un problema, se identifican las necesidades del aprendizaje, se busca la información necesaria para solucionarlo y, finalmente, se resuelve.

PALABRAS CLAVE: Aprendizaje - Competencias - Educación Superior Pluridisciplinar

\section{HOW TO OBTAIN SPECIFIC SKILLS AND CROSS FROM THEMASS MEDIA. ORIGINAL APPLI CATION OF APP IN UDG}

\begin{abstract}
This article presents a project that includes APP mutidisciplinardisciplines are as diverse as IT, Engineering, Physics and the public and allows to acquire specific skills of each subject, and develop common generic skills such as teamwork, creative design proposals or communicate orally and in writing. The project has the originality of mixing in the same segments workvarious university students who otherwise would work hardconnected, thereby fostering creativity in a scientific environment. The project's goal is to transform a television program Arguiñano Karlos on a radio program. To do this students must identify, analyze and transform the various scientific, technical and communication are seen on the television program so that it canturn into radio. The choice of this axis of APP is the fruit of long reflection to find a

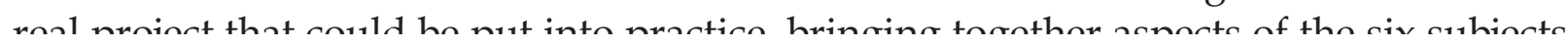


in different fields of knowledge involved in this network of educational innovation. The implementation of the APP, increasingly used in college, is aprocess in which there is a problem, identify learning needs, we seek the information needed to solve it and finally resolved.

KEY WORDS: Learning - Qualifications - Higher education - Multidisciplinary

\section{INTRODUCCIÓN}

Este proyecto de APP ha sido bautizado como proyecto Arguiñano y ha sido diseñado por siete profesores de la Universidad de Girona, miembros de la Red de Innovación Docente de Aprendizaje por Proyectos (APP) del Instituto de Ciencias de la Educación (ICE) Josep Pallach, procedentes de ámbitos de conocimiento tan diferentes como informática, ingeniería, física o publicidad y relaciones públicas. Actualmente, el proyecto Arguiñano está totalmente definido y se va a implementar a partir de febrero del 2012.

El proyecto tiene como objetivo explicar la ciencia que se oculta en el programa de televisión de Karlos Arguiñano y que está relacionado con cada una de las asignaturas implicadas en este equipo de trabajo que suma conocimientos tan dispares (Figura 1). Una vez identificada la ciencia de la cocina de Arguiñano, se explicará a través de un programa de radio. La elección de éste espacio culinario como eje vertebrador del APP es fruto de una larga reflexión, cuya finalidad fue encontrar una idea muy conectada con la realidad, que se pudiera llevar a la práctica y que aglutinara las seis asignatura de los diferentes ámbitos de conocimiento implicados; intentando que las disciplinas autónomas perdieran sólo parcialmente su carácter para formar parte de una unidad más global.

Esta idea integradora permite que los estudiantes de comunicación se den cuenta de la importancia que tiene dotar de contenido contrastado científicamente un programa de radio. Mientras que, por otro lado, los estudiantes de un perfil más científicotecnológico aprenden a transmitir sus conocimientos usando un lenguaje menos específico y de forma más divulgativa y dinámica. Ambos enfoques no son para nada contradictorios y parece importante manifestarlo en un entorno como éste. Los profesionales de la comunicación sabemos que es posible y que las técnicas propias de las disciplinas de nuestro campo son básicas, entre otras cosas, para crear buenas sinergias de comunicación para cualquier ámbito. 


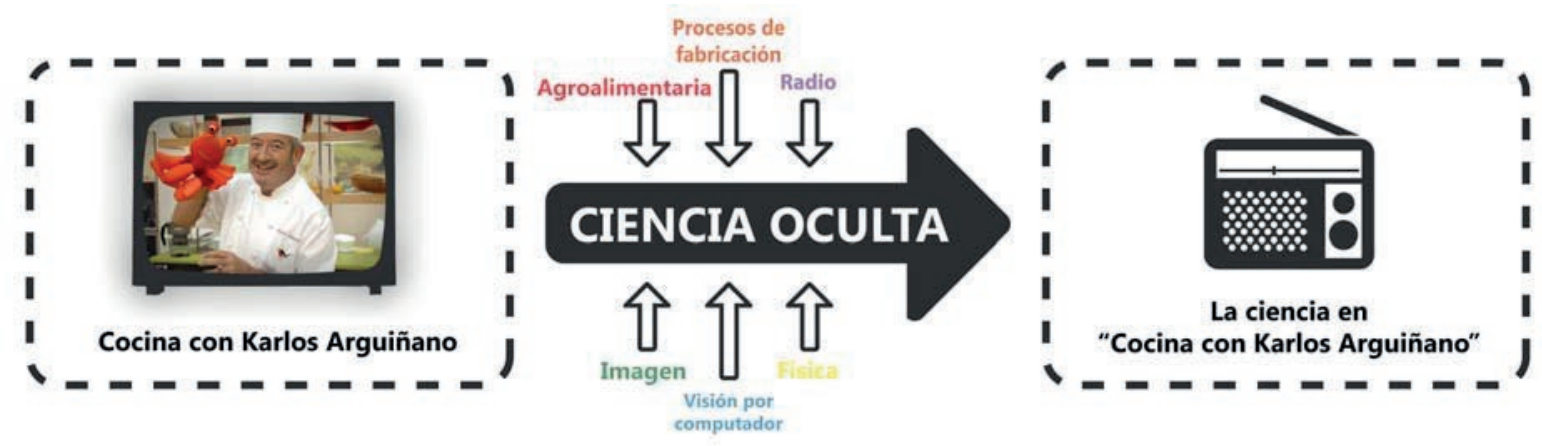

Figura 1. Objetivo del proyecto Arguiñano. Fuente: Elaboración propia

Uno de los principales retos planteados por el equipo multidisciplinar de profesores fue diseñar un plan suficientemente transversal que permitiera, de forma simultánea, desarrollar competencias específicas, propias de cada asignatura y competencias transversales, comunes a todas ellas. Entre las diferentes competencias transversales que se pudieran desarrollar en un proyecto fundamentado en el aprendizaje autónomo y compartido se consideraron: trabajar en equipo, diseñar propuestas creativas, comunicarse oralmente y por escrito, y utilizar tecnologías de la información y la comunicación.

\section{METODOLOGÍA}

\subsection{El método de aprendizaje por proyectos}

El aprendizaje por proyectos (APP) es uno de los métodos renovadores del proceso de enseñanza-aprendizaje que más se está consolidando en las instituciones de educación superior últimamente. En el caso del APP, primero se plantea un propósito, después se identifican las necesidades de aprendizaje que conlleva, luego se busca la información necesaria para desarrollarlo $y$, finalmente, se elabora el proyecto.

Durante el desarrollo del proyecto los estudiantes trabajan cooperativamente en pequeños grupos desde el planteamiento original hasta la consecución de la solución, compartiendo en esta experiencia de aprendizaje la práctica y el desarrollo de habilidades relacionadas con competencias específicas y transversales, así como también la posibilidad de observar y reflexionar sobre actitudes y valores que con el método convencional expositivo difícilmente se pueden poner en acción (Valero, 2007).

Existen antecedentes en la aplicación del APP en agrupaciones de materias de un mismo nivel dentro de un plan de estudios de forma que dichas materias se conviertan en un único bloque docente (Anguas et al., 2006) pero también en la 
Las experiencias que se tienen en este ámbito indican que, efectivamente, en los últimos cursos, los alumnos ya tienen un buen entrenamiento en la metodología y aprenden mucho más rápido y mejor, compensando con creces las reducciones de temarios que hayan sido necesarios en los primeros cursos. En planes de estudios totalmente basados en APP es habitual que los rendimientos académicos sean muy superiores a los que se obtienen con planes tradicionales, especialmente en las ingenierías (Alcober et al., 2003). Sin embargo, no se han encontrado en la bibliografía experiencias de APP conjuntas para ámbitos de conocimiento tan dispares como los que aquí se presentan.

Vivimos en un momento social y en un entorno cultural donde el ciudadano puede, además de consumir, producir información a partir de la universalización de la tecnología, y casi inmediatamente, difundirla a través de multitud de redes y plataformas sociales. Si nuestros alumnos pueden realizar videoreportajes caseros o programas de radio y colgarlos en la red (podcast), es lógico que piensen y nos planteen por qué en nuestras facultades hay que aleccionarles con el aprendizaje basado en el método que podríamos denominar unilateral y unívoco.

Con el proyecto Arguiñano responderemos a este planteamiento y demostraremos cómo realizar programas de radio juveniles, con contenidos rigurosos, a partir de un programa de televisión de fácil descodificación pero que utiliza la ciencia como base de su existencia. Los argumentos que surgen de disciplinas como la física o la robótica se pueden convertir en contenidos contrastados y ser comunicados con un lenguaje interesante, el radiofónico, que se adapta al uso que hacen nuestros alumnos de las nuevas tecnologías.

El proyecto Arguiñano servirá pues, y parafraseando a Marina (2010), para resolver los problemas y avanzar con las soluciones, "incluyendo la idea de excelencia, de logro y de eficacia. Puesto que hay muchas inteligencias diferentes, puede haber muchos talentos distintos, cada uno de los cuales supone un especial tipo de destreza".

\section{ANÁLISIS Y DISCUSIÓN}

\subsection{Descripción del proyecto}

\section{Propuesta}

Como ya se ha mencionado anteriormente, el proyecto Arguiñano se aplica conjuntamente en asignaturas de ámbitos diversos, de planes de estudios distintos y se involucran alumnos de cursos diferentes. Incluyendo estudiantes de primer curso de diversos grados de Ingeniería, estudiantes de segundo curso del Grado en Publicidad y Relaciones Públicas y alumnos del Máster en Visión por Computador y Robótica. Ninguno de ellos ha trabajado anteriormente con el modelo de aprendizaje 
La Tabla 1 muestra las distintas asignaturas y estudios implicados, así como las competencias transversales que se desarrollan en el proyecto y las específicas de cada disciplina.

Tabla 1. Estudios, asignaturas, créditos ECTS asignados, y competencias transversales y específicas del proyecto Arguiñano.

\begin{tabular}{|c|c|c|c|c|}
\hline Estudio & Asignatura & ECTS & $\begin{array}{l}\text { Competencias } \\
\text { transversales }\end{array}$ & $\begin{array}{c}\text { Competencias } \\
\text { específicas }\end{array}$ \\
\hline \multirow[t]{2}{*}{$\begin{array}{l}\text { Grado en } \\
\text { Publicidad y } \\
\text { Relaciones } \\
\text { Públicas }\end{array}$} & $\begin{array}{l}\text { Teoría de la } \\
\text { Imagen }\end{array}$ & 2 & \multirow[t]{2}{*}{$\begin{array}{l}\text { СT02. } \\
\text { Comunicarse } \\
\text { oralmente y por } \\
\text { escrito. } \\
\text { CT03. Utilizar } \\
\text { tecnologías de la } \\
\text { información y la } \\
\text { comunicación }\end{array}$} & $\begin{array}{l}\text { M7-4. Aplicar las técnicas y } \\
\text { Procesos de difusión } \\
\text { audiovisuales en sus diversas } \\
\text { fases, tanto en los medios } \\
\text { tradicionales como en los medios } \\
\text { hipermedia, desde el punto de } \\
\text { vista de la puesta en escena de la } \\
\text { idea hasta su acabado final. } \\
\text { M7-7. Analizar y aplicar los } \\
\text { fundamentos de la sintaxis de la } \\
\text { imagen en el diseño estratégico } \\
\text { de un icono. }\end{array}$ \\
\hline & \begin{tabular}{l}
\multicolumn{1}{c}{ Teoría y } \\
Técnica del \\
Lenguaje \\
Radiofónico
\end{tabular} & 5 & & $\begin{array}{l}\text { M7-8. Comunicar en lenguaje } \\
\text { propio de cada uno de los } \\
\text { medios tradicionales, en sus } \\
\text { formas combinadas (multimedia) } \\
\text { o soportes digitales. }\end{array}$ \\
\hline
\end{tabular}




\begin{tabular}{|c|c|c|c|c|}
\hline & & & $\begin{array}{l}\text { CT06. Diseñar } \\
\text { propuestas } \\
\text { creativas }\end{array}$ & $\begin{array}{l}\text { M7-9. Producir y realizar un } \\
\text { producto audiovisual de ficción. } \\
\text { M7-10. Leer, comprender y } \\
\text { comentar textos científicos }\end{array}$ \\
\hline $\begin{array}{l}\text { Máster en Visión } \\
\text { por Computador y } \\
\text { Robótica }\end{array}$ & $\begin{array}{l}\text { Percepción } \\
\text { Visual }\end{array}$ & 6 & & $\begin{array}{l}\text { CE01. Comprender el } \\
\text { funcionamiento y las limitaciones } \\
\text { de los invariant features } \\
\text { CE02. Identificar, valorar y } \\
\text { utilizar técnicas y métodos } \\
\text { actuales y novedosos para la } \\
\text { resolución de proyectos } \\
\text { tecnológicos y de investigación } \\
\text { en la visión por computador y } \\
\text { robótica, la ingeniería de control } \\
\text { y los sistemas inteligentes. }\end{array}$ \\
\hline $\begin{array}{l}\text { Grados en } \\
\text { Ingeniería } \\
\text { Electrónica, } \\
\text { Eléctrica, Mecánica } \\
\text { y Química }\end{array}$ & $\begin{array}{c}\text { Fundamentos } \\
\text { Físicos de la } \\
\text { Ingeniería }\end{array}$ & 2 & & $\begin{array}{l}\text { CE03. Comprensión y dominio } \\
\text { de los conceptos básicos sobre las } \\
\text { leyes de la termodinámica y } \\
\text { ondas, y su aplicación. }\end{array}$ \\
\hline $\begin{array}{l}\text { Grado en } \\
\text { Ingeniería } \\
\text { Mecánica }\end{array}$ & $\begin{array}{c}\text { Procesos de } \\
\text { Fabricación } 2\end{array}$ & 1 & & $\begin{array}{l}\text { CE28. Conocimiento aplicado de } \\
\text { los sistemas y procesos de } \\
\text { fabricación, metrología y control } \\
\text { de calidad. }\end{array}$ \\
\hline $\begin{array}{l}\text { Grado en } \\
\text { Ingeniería } \\
\text { Agroalimentaria }\end{array}$ & Proyectos & 1,5 & & $\begin{array}{l}\text { CE15. Capacidad para conocer, } \\
\text { comprender y utilizarlos } \\
\text { principios de los proyectos } \\
\text { técnicos. } \\
\text { CE18. Capacidad para conocer, } \\
\text { comprender y utilizarlos } \\
\text { principios de transferencia de } \\
\text { tecnología. }\end{array}$ \\
\hline
\end{tabular}


El proyecto Arguiñano se despliega en dos partes claramente diferenciadas: la específica y la transversal. De este modo, el desarrollo del propósito conlleva la aplicación de especialidades propias de cada una de las materias que intervienen.

a) La parte específica tiene una duración diferente en cada materia en función de la carga crediticia de cada asignatura implicada (Tabla 1), aunque no debería durar más de 5 semanas. En esta parte los alumnos de cada asignatura deben elaborar un trabajo para identificar los aspectos científicos del programa de televisión que están relacionados con su disciplina, asumiendo así las competencias específicas asignadas a su materia. Este trabajo se desarrolla en grupos de tres alumnos que se convierten en los expertos de su ámbito.

b) La parte transversal tiene una duración de tres semanas. En ella los alumnos expertos deben compartir y transmitir el conocimiento específico a los expertos de los otros ámbitos con el objetivo de elaborar un guión radiofónico que comunique la ciencia oculta relacionada con las diferentes disciplinas al público radioyente, de una forma clara, atractiva y de calidad. En esta parte los alumnos se convierten en productores del programa de radio y por consiguiente se les denominará así durante el resto del proyecto.

El planteamiento del proyecto Arguiñano usando el APP pretende que los alumnos sean más responsables de su trabajo y de su proceso de aprendizaje, de acuerdo con Benito Capa y Cruz Chust (2007). Los estudiantes adquieren unadependencia positiva entre ellos para alcanzar con éxito los objetivos propuestos. Simultáneamente se facilita una mejora del rendimiento académico. Tuning en 2000 ya aconsejaba planificar asignaturas para promocionar el alto rendimiento de los estudiantes (Yáñiz Álvarez y Villardón Gallego, 2006). La mejora del rendimiento académico es favorecida por un aumento de la comunicación entre el alumno y el profesor pues el feed-back entre profesores y alumnos se vuelve más fluido debido a que el profesor adquiere un rol de guía del proceso de aprendizaje del alumno.

\subsection{Creación de grupos}

Como se ha mencionado en la sección anterior, durante el desarrollo del trabajo se forman dos tipos de grupos: el "grupo de alumnos expertos" y el "grupo de alumnos productores" (Figura 2). En primer lugar se constituyen los "grupos de expertos" dentro de cada ámbito de conocimiento; los cuales están formados por tres alumnos de una misma disciplina. Este tipo de equipo tiene un carácter homogéneo y opera durante la fase específica del proyecto. 
Posteriormente, para formar el "grupo de productores", se integra un experto de cada ámbito de conocimiento en un grupo productor, de forma que este grupo pueda disponer de todo el conocimiento específico necesario para poder elaborar el programa final. Este proceso de agrupación está ejemplificado mediante colores en la Figura 2. Cada "grupo de expertos" está representado con un color, indicando de ese modo cada uno de los ámbitos de conocimiento que participan del proyecto (un total de 6 colores diferentes). Por otro lado, cada "grupo de productores" contiene un miembro experto de cada disciplina específica, de forma que todos los colores están incluidos en este tipo de grupo. La formación de los grupos de expertos se deja a libertad de los alumnos. En el diseño también se contempla el papel del grupo de formadores o equipo docente que actúan como facilitadores acompañando en el aprendizaje.
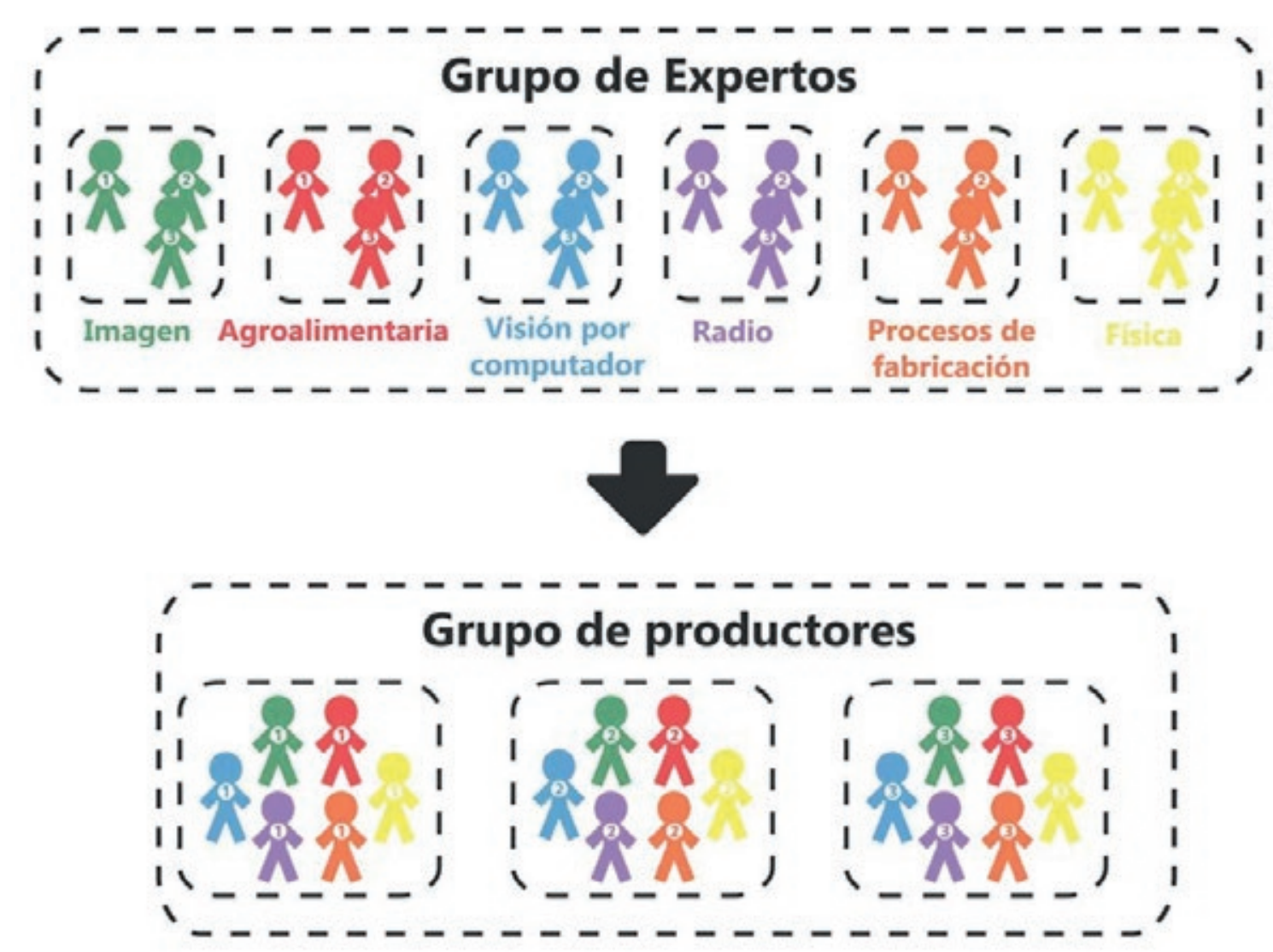

Figura 2. Representación de grupos de expertos y grupos de producción. Fuente: Elaboración propia 


\subsection{Fases de implementación}

En esta experiencia, el proyecto se inicia con un programa concreto de cocina de Karlos Arguiñano. Este programa permite formular la pregunta clave, eje vertebrador de toda la idea: ¿Cuál es la ciencia que está oculta dentro del programa de Arguiñano? La segunda fase consiste en delimitar el problema. Para responder una pregunta tan genérica como la planteada es necesario reorientar a los alumnos en el proceso de búsqueda de la solución en dos vertientes: una hacia su materia específica y otra con más transversalidad. Así, pues, al alumnado participante se le formulan algunas preguntas orientativas para reconducirlo hacia la pregunta clave.

Una vez formuladas las preguntas, el alumno debe elaborar mapas conceptuales a partir de los conocimientos que necesitará para responder a estas preguntas con la intención de poner de manifiesto lo que se conoce y lo que no. Esto pone de relieve cuáles son los conocimientos de partida y cuáles son las carencias. En la experiencia se le da al alumno una guía didáctica o plan de trabajo que incluye toda la documentación necesaria para que pueda llevar a cabo su propio proceso de aprendizaje, y también le permite saber en todo momento de que será evaluado y cómo será evaluado (Buck Institute for Education, 2003).

En la tercera fase se delimitan los recursos y las acciones, es decir, se planifica el proyecto. En este sentido, el equipo de profesores diseña un conjunto de actividades tanto individuales como colectivas. Algunas de estas actividades son evaluadas y otras, simplemente, sirven para orientar al alumno en su autoaprendizaje (Escoda i Acero et al., 2009).

La cuarta fase corresponde al trabajo individual. Este apartado hace referencia tanto a las horas de estudio individual del alumno como a la elaboración de aquellas tareas individuales que están previstas en el diseño del plan. Finalmente, la quinta fase corresponde al trabajo en equipo. En la presente experiencia, la cuarta y la quinta fase se llevarán a cabo de forma simultánea.

Son muchas las cuestiones a considerar en el diseño de un macro bloque docente basado en proyectos y que pueden ser la clave del éxito de éste. Se hace referencia aquí sólo a los aspectos que se consideran más importantes como son, el establecimiento de una guía o plan de trabajo y la conveniencia de un buen plan de seguimiento del proyecto. En el plan de seguimiento, que será semanal, se incluyen entregas frecuentes de resultados parciales que son utilizados por el equipo docente para informar puntualmente a los alumnos del grado de consecución de las competencias o para determinar acciones complementarias que permitan el logro de las mismas. 


\subsection{Planificación}

El proyecto Arguiñano está diseñado para ser realizado en una totalidad de siete semanas. En la primera semana se presenta el proyecto a todos los alumnos participantes, se realiza una sesión expositiva de los aspectos específicos a desarrollar por parte de cada profesor y, posteriormente, se forman los grupos. Los estudiantes visionan un DVD del programa de cocina de Karlos Arguiñano que se va a trabajar utilizando una lista de comprobación (checklist) particularizada para cada asignatura.

De la semana dos a la semana cuatro cada disciplina planifica: (i) las actividades específicas identificando la tipología de cada una de ellas (actividades presenciales, no presenciales, autónomas y colectivas), (ii) los recursos necesarios para llevar a cabo la tarea y (iii) los entregables resultantes para la evaluación. La planificación esta parte específica para cada una de las disciplinas se expone desde la Tabla 2 hasta la Tabla 7. Como se puede observar en todas estas tablas, en las últimas semanas de cada grupo específico se debe entregar un informe final del trabajo realizado, al profesor responsable, con los contenidos específicos necesarios para poder elaborar el programa de radio. 
Tabla 1. Planificación de la disciplina: Física. Nomenclatura: CP-Con profesor; G/SPGrupo/sin profesor

\begin{tabular}{ccccccc}
\hline Semana & Tarea CP & Horas & Tarea G/SP & Horas & Recursos & Entregable \\
\hline $\mathbf{1}$ & $\begin{array}{c}\text { Presentación y } \\
\text { definición del } \\
\text { proyecto. } \\
\text { Sesión }\end{array}$ & 0,5 & Visionar DVD & 2 & $\begin{array}{c}\text { Ficha de } \\
\text { conceptos } \\
\text { básicos }\end{array}$ & $\begin{array}{c}\text { Acta de } \\
\text { reuniones. }\end{array}$ \\
& 1 & Preparación de & 3 & DVD del & Esquema de
\end{tabular}

\begin{tabular}{|c|c|c|c|c|c|c|}
\hline & $\begin{array}{l}\text { expositiva: } \\
\text { Conceptos } \\
\text { básicos } \\
\text { Tutoría (E1) }\end{array}$ & 1 & $\begin{array}{l}\text { un "Esquema } \\
\text { del trabajo" }\end{array}$ & & programa & trabajo $(\mathrm{E} 1)$ \\
\hline 2 & $\begin{array}{l}\text { Sesión } \\
\text { expositiva: } \\
\text { Conceptos } \\
\text { básicos }\end{array}$ & 1 & $\begin{array}{l}\text { Elaboración del } \\
\text { “contenido } \\
\text { científico del } \\
\text { programa" (E2). } \\
\text { Corrección del } \\
\text { E1 }\end{array}$ & 2,5 & & \\
\hline \multirow[t]{2}{*}{3} & $\begin{array}{l}\text { Sesión } \\
\text { Expositiva: } \\
\text { Conceptos } \\
\text { básicos y } \\
\text { problemas } \\
\text { relacionados }\end{array}$ & 1 & $\begin{array}{l}\text { Elaboración del } \\
\text { E2 }\end{array}$ & 2 & $\begin{array}{l}\text { Dossier de } \\
\text { problemas } \\
\text { de carácter } \\
\text { individual } \\
\text { (E3i) y } \\
\text { grupal (E3) }\end{array}$ & $\begin{array}{c}\text { Actas de la } \\
\text { reuniones } \\
\text { E1 } \\
\text { definitivo }\end{array}$ \\
\hline & $\begin{array}{l}\text { Tutoría (E2 + } \\
\text { E1corregido) }\end{array}$ & 1 & $\begin{array}{c}\text { Preparación del } \\
\text { E3 (grupo) y E3i } \\
\text { (individual) }\end{array}$ & 2 & & $\begin{array}{l}\text { Contenido } \\
\text { científico } \\
\text { del } \\
\text { programa } \\
\text { (E2) }\end{array}$ \\
\hline 4 & & & $\begin{array}{l}\text { Elaboración del } \\
\text { E3 y corrección } \\
\text { del E2 }\end{array}$ & 1 & & $\begin{array}{c}\text { Actas de las } \\
\text { reuniones } \\
\text { E2 } \\
\text { definitivo }\end{array}$ \\
\hline \multirow[t]{2}{*}{5} & $\begin{array}{l}\text { Tutoría (E3i, E3 } \\
\text { y E2 corregido) }\end{array}$ & 1 & $\begin{array}{c}\text { Corrección del } \\
\text { E3 (grupo) y E3i } \\
\text { (indv.) }\end{array}$ & 1 & & $\begin{array}{c}\text { Actas de las } \\
\text { reuniones }\end{array}$ \\
\hline & $\begin{array}{c}\text { Tutoría (E3i, E3 } \\
\text { corregido y } \\
\text { Exposición oral } \\
\text { parte científica) }\end{array}$ & 1,5 & $\begin{array}{c}\text { Preparación de } \\
\text { la exposición } \\
\text { oral del trabajo } \\
\text { específico }\end{array}$ & 1,5 & & $\begin{array}{c}\text { E3 y E3i } \\
\text { definitivo }\end{array}$ \\
\hline
\end{tabular}


Tabla 2. Planificación de la disciplina: Teoría de la Imagen. Nomenclatura: CP-Con profesor; G/SP-Grupo/sin profesor

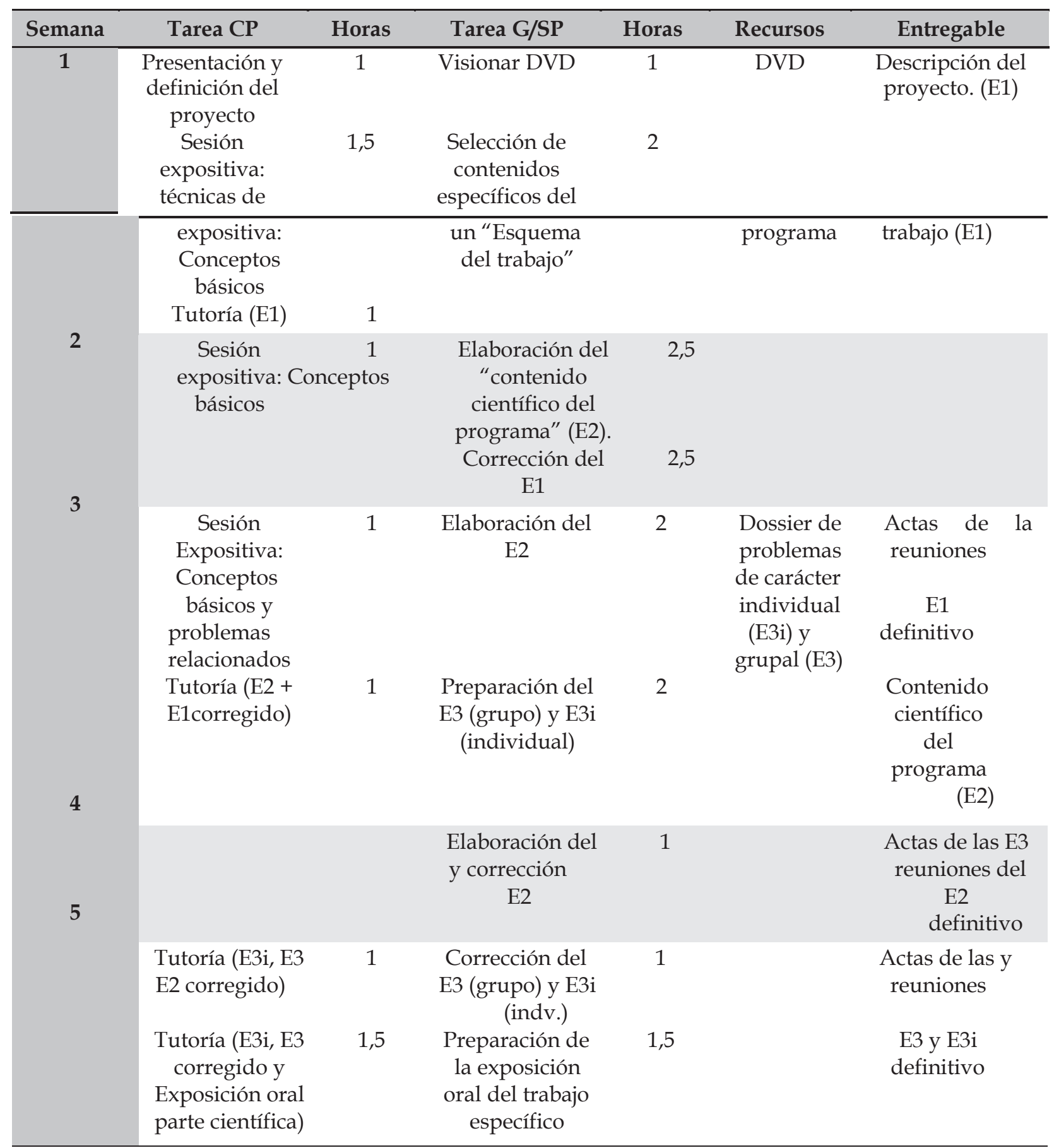


Tabla 3. Planificación de la disciplina: Procesos de fabricación. Nomenclatura: CP-Con profesor; G/SP-Grupo/sin profesor

\begin{tabular}{|c|c|c|c|c|c|c|}
\hline $\begin{array}{l}\text { Seman } \\
\text { a }\end{array}$ & Tarea CP & Horas & Tarea G/SP & Horas & Recursos & Entregable \\
\hline \multirow[t]{2}{*}{1} & $\begin{array}{l}\text { Presentación } \\
\text { del proyecto }\end{array}$ & 1 & Visionar DVD & 0,5 & \multirow[t]{2}{*}{ DVD } & \multirow[t]{2}{*}{$\begin{array}{l}\text { Lista elementos } \\
\text { (E1) }\end{array}$} \\
\hline & Tutoría (E1) & 1 & $\begin{array}{l}\text { Selección } \\
\text { utensilios }\end{array}$ & 1 & & \\
\hline \multirow[t]{2}{*}{2} & $\begin{array}{l}\text { Sesión } \\
\text { expositiva }\end{array}$ & 2 & $\begin{array}{c}\text { Identificar } \\
\text { posibles cadenas } \\
\text { de procesos y } \\
\text { seleccionar }\end{array}$ & 2,5 & \multirow[t]{2}{*}{ Internet } & \multirow[t]{2}{*}{$\begin{array}{l}\text { Lista de } \\
\text { requerimientos } \\
\text { y restricciones } \\
\quad(\mathrm{E} 2)\end{array}$} \\
\hline & & & $\begin{array}{c}\text { Analizar } \\
\text { requerimientos }\end{array}$ & 1,5 & & \\
\hline \multirow[t]{2}{*}{3} & & & $\begin{array}{c}\text { Investigar el } \\
\text { material, } \\
\text { determinar } \\
\text { parámetros del }\end{array}$ & \multirow[t]{2}{*}{2} & Bibliografía & \\
\hline & & & $\begin{array}{l}\text { proceso y } \\
\text { máquinas }\end{array}$ & & & \\
\hline \multirow[t]{2}{*}{4} & & & $\begin{array}{c}\text { Elaboración del } \\
\text { trabajo final }\end{array}$ & \multirow[t]{2}{*}{4} & & $\begin{array}{c}\text { Contenido } \\
\text { científico (E3) }\end{array}$ \\
\hline & Tutoría (E2) & 2 & & & & $\begin{array}{c}\text { Trabajo } \\
\text { específico (E4) }\end{array}$ \\
\hline
\end{tabular}


Tabla 4. Planificación de la disciplina: Proyectos agroalimentarios. Nomenclatura: CPCon profesor; G/SP-Grupo/sin profesor

\begin{tabular}{|c|c|c|c|c|c|c|}
\hline Semana & Tarea CP & Horas & Tarea G/SP & Horas & Recursos & Entregable \\
\hline 1 & $\begin{array}{l}\text { Presentación } \\
\text { del proyecto y } \\
\text { visionado del } \\
\text { DVD }\end{array}$ & 1 & $\begin{array}{c}\text { Intercambio de } \\
\text { opiniones } \\
\text { sobre el } \\
\text { programa } \\
\text { Definición del } \\
\text { plan de trabajo } \\
\text { Identificación } \\
\text { de la } \\
\text { información a } \\
\text { buscar. Reparto } \\
\text { de tareas }\end{array}$ & 1,5 & \begin{tabular}{l}
\multicolumn{1}{c}{ DVD } \\
Documento \\
de plantea- \\
miento del \\
proyecto
\end{tabular} & $\begin{array}{c}\text { Acta de la } \\
\text { reunión y plan } \\
\text { de trabajo (E1) }\end{array}$ \\
\hline 2 & $\begin{array}{c}\text { Sesión } \\
\text { expositiva }\end{array}$ & 1 & $\begin{array}{c}\text { Discusión del } \\
\text { programa } \\
\text { Identificación } \\
\text { de las } \\
\text { alternativas de } \\
\text { materias } \\
\text { primeras, } \\
\text { procesos } \\
\text { productivos y } \\
\text { comercializació } \\
n \\
\text { Evaluación y } \\
\text { elección de las } \\
\text { alternativas }\end{array}$ & $\begin{array}{c}0,5 \\
3\end{array}$ & $\begin{array}{l}\text { Criterios de } \\
\text { presenta- } \\
\text { ción } \\
\text { Listados de } \\
\text { verificación } \\
\text { Bibliografía } \\
\text { Internet }\end{array}$ & $\begin{array}{c}\text { Acta de la } \\
\text { reunión y anejo } \\
\text { de estudio de } \\
\text { las alternativas } \\
\text { (E2) }\end{array}$ \\
\hline 3 & $\begin{array}{l}\text { Tutoría y } \\
\text { corrección del } \\
\text { anejo de } \\
\text { estudio de } \\
\text { alternativas } \\
\text { (E2) }\end{array}$ & 1 & $\begin{array}{c}\text { Definición y } \\
\text { dimensionamie } \\
\text { nto del plan } \\
\text { productivo } \\
\text { Identificación } \\
\text { de las } \\
\text { necesidades del } \\
\text { plan } \\
\text { productivo }\end{array}$ & 1 & $\begin{array}{l}\text { Bibliografía } \\
\text { Internet }\end{array}$ & $\begin{array}{c}\text { Acta de la } \\
\text { reunión y } \\
\text { anexo de } \\
\text { ingeniería del } \\
\text { proceso (E3) }\end{array}$ \\
\hline
\end{tabular}

\begin{tabular}{|c|c|c|c|c|c|c|}
\hline 4 & $\begin{array}{c}\text { Tutoría y } \\
\text { corrección del } \\
\text { anejo de } \\
\text { ingeniería del } \\
\text { proceso (E3) }\end{array}$ & 1 & $\begin{array}{c}\text { Dimensionami } \\
\text { ento preliminar } \\
\text { de la industria } \\
\text { transformadora } \\
\text { Estudio de } \\
\text { viabilidad } \\
\text { económica } \\
\text { Identificación } \\
\text { de los aspectos } \\
\text { a explicar en el }\end{array}$ & $\begin{array}{l}2 \\
1\end{array}$ & $\begin{array}{c}\text { Bibliografía } \\
\text { Internet }\end{array}$ & $\begin{array}{c}\text { Acta de la } \\
\text { reunión y } \\
\text { anteproyecto } \\
\text { de la industria } \\
\text { transforma- } \\
\text { dora (E4) }\end{array}$ \\
\hline
\end{tabular}


Tabla 5. Planificación de la disciplina: Percepción Visual. Nomenclatura: CP-Con profesor; G/SP-Grupo/sin profesor

\begin{tabular}{|c|c|c|c|c|c|c|}
\hline Semana & Tarea CP & Horas & Tarea/SP & Horas & Recursos & Entregable \\
\hline \multirow[t]{2}{*}{1} & $\begin{array}{l}\text { Presentación del } \\
\text { proyecto }\end{array}$ & 1 & Visionar DVD & 0,5 & DVD & $\begin{array}{c}\text { Descripción del } \\
\text { proyecto (E1) }\end{array}$ \\
\hline & Tutoría (E1) & 1 & $\begin{array}{l}\text { Definición de cómo } \\
\text { se va a llevar a cabo } \\
\text { el proyecto: ¿cuál es } \\
\text { el objeto a } \\
\text { reconocer? (logos, } \\
\text { utensilios, etc.) }\end{array}$ & 1 & & \\
\hline 2 & $\begin{array}{l}\text { Análisis teórico } \\
\text { de posibles } \\
\text { soluciones }\end{array}$ & 2 & $\begin{array}{l}\text { Búsqueda de } \\
\text { información sobre } \\
\text { reconocimiento de } \\
\text { patrones } \\
\text { Seleccionar posibles } \\
\text { técnicas Búsqueda } \\
\text { de código ejecutable } \\
\text { para las técnicas } \\
\text { seleccionadas }\end{array}$ & $\begin{array}{c}1 \\
2,5\end{array}$ & Internet & $\begin{array}{l}\text { Lista de técnicas de } \\
\text { reconocimiento de } \\
\text { patrones } \\
\text { potencialmente } \\
\text { utilizables en el } \\
\text { proyecto (E2) }\end{array}$ \\
\hline 3 & $\begin{array}{l}\text { Verificación del } \\
\text { funcionamiento } \\
\text { del código }\end{array}$ & 1 & $\begin{array}{c}\text { Pruebas de } \\
\text { funcionamiento del } \\
\text { código disponible / } \\
\text { pequeñas } \\
\text { implementaciones } \\
\text { en Matlab }\end{array}$ & 4 & $\begin{array}{c}\text { Matlab, } \\
\text { código } \\
\text { descargado } \\
\text { de internet }\end{array}$ & $\begin{array}{c}\text { Código } \\
\text { fuente/ ejecutables } \\
\text { (E3) }\end{array}$ \\
\hline 4 & Tutoría (E2) & 2 & $\begin{array}{l}\text { Elaboración del } \\
\text { trabajo final }\end{array}$ & 3 & & $\begin{array}{c}\text { Trabajo específico } \\
\text { (E4) }\end{array}$ \\
\hline
\end{tabular}


Tabla 6. Planificación de la disciplina: Lenguaje radiofónico. Nomenclatura: $\mathrm{CP}-\mathrm{Con}$ profesor; G/SP- Grupo/sin profesor

\begin{tabular}{|c|c|c|c|c|c|c|}
\hline Semana & Tarea CP & Horas & Tarea /SP & Horas & Recursos & Entregable \\
\hline 1 & $\begin{array}{l}\text { Presentación } \\
\text { del proyecto } \\
\text { Sesión } \\
\text { expositiva }\end{array}$ & 1 & $\begin{array}{l}\text { Visionar DVD } \\
\text { Identificar } \\
\text { convertibles }\end{array}$ & 5 & $\begin{array}{c}\text { DVD } \\
\text { Guía de } \\
\text { visionado }\end{array}$ & $\begin{array}{c}\text { Acta } \\
\text { Trabajo de } \\
\text { identificación de } \\
\text { convertibles }\end{array}$ \\
\hline 2 & $\begin{array}{l}\text { Sesión } \\
\text { expositiva }\end{array}$ & 1 & $\begin{array}{l}\text { Visionado de } \\
\text { modelos }\end{array}$ & 4 & $\begin{array}{l}\text { Programas/ } \\
\text { modelo } \\
\text { Ejemplos de } \\
\text { redacción de } \\
\text { textos y } \\
\text { guiones }\end{array}$ & \\
\hline 3 & $\begin{array}{c}\text { Sesión } \\
\text { expositiva }\end{array}$ & 1 & $\begin{array}{c}\text { Selección de } \\
\text { modelos con } \\
\text { estructura y géneros } \\
\text { identificados }\end{array}$ & 2 & $\begin{array}{l}\text { Modelos } \\
\text { elegidos }\end{array}$ & $\begin{array}{c}\text { Acta } \\
1^{\text {a }} \text { pauta }\end{array}$ \\
\hline 4 & $\begin{array}{l}\text { Revisión } \\
\text { pauta } \\
\text { Expositiva }\end{array}$ & 2 & $\begin{array}{l}\text { Construir } \\
\text { transiciones a la } \\
\text { textualidad } \\
\text { radiofónica }\end{array}$ & 3 & & $\begin{array}{c}\text { Acta } \\
\text { Pauta/Guión } \\
\text { literario } 1^{\circ}\end{array}$ \\
\hline
\end{tabular}

La Tabla 7 muestra la planificación correspondiente a las últimas tres semanas del proyecto, en las que trabajan los grupos productores con el material elaborado en la fase anterior y la cuál es liderada por la disciplina de Lenguaje radiofónico. Como se muestra en la Tabla 6, entre las semanas 5 y 7, el recurso básico para empezar esta fase son los trabajos específicos de cada disciplina que llegan en forma de trabajo escrito y que se irán modelando a partir de las tutorías de revisión pautadas para convertirse en formatos sonoros. Al final del período cada grupo de productores debe entregar un guión final, escrito, del programa de radio, y una maqueta definitiva.

Finalmente, en la última semana, se hacen públicas las maquetas en la intranet docente y se abre el plazo para que los alumnos productores co-evalúen y los profesores las evalúen respectivamente, mediante rúbricas elaboradas por el equipo docente. 
Tabla 7. Planificación del trabajo interdisciplinar. Las 3 últimas semanas de Proyecto Arguiñano. Nomenclatura: CP-Con profesor; G/SP-Grupo/sin profesor

\begin{tabular}{|c|c|c|c|c|c|c|}
\hline Semana & Tarea CP & Horas & Tarea G/SP & Horas & Recursos & Entregable \\
\hline 5 & $\begin{array}{c}\text { Sesión } \\
\text { expositiva }\end{array}$ & 1 & $\begin{array}{c}\text { Intercambio de } \\
\text { opiniones a } \\
\text { partir de sesión } \\
\text { dada. } \\
\text { Modificaciones } \\
\text { sobre el } \\
\text { proyecto } \\
\text { presentado. }\end{array}$ & 2 & & $\begin{array}{l}\text { Redacción de } \\
\text { contenidos } \\
\text { científicos para } \\
\text { el guión }\end{array}$ \\
\hline 6 & $\begin{array}{l}\text { Tutoría. } \\
\text { Corrección del } \\
\text { guión y pautas } \\
\text { de locución. }\end{array}$ & 2 & $\begin{array}{c}\text { Redacción del } \\
\text { guión } \\
\text { definitivo. } \\
\text { "Congelamos" } \\
\text { Grabación de la } \\
\text { primera } \\
\text { maqueta }\end{array}$ & 4 & TICS/sonido & $1^{\mathrm{a}}$ maqueta \\
\hline 7 & $\begin{array}{l}\text { Tutoría. } \\
\text { Revisión } 1^{\mathrm{a}} \\
\text { maqueta. } \\
\text { Análisis. }\end{array}$ & 2 & $\begin{array}{l}\text { Re-elaboración } \\
\text { de la maqueta }\end{array}$ & 8 & & $\begin{array}{l}\text { Maqueta } \\
\text { definitiva }\end{array}$ \\
\hline
\end{tabular}

\subsection{Evaluación}

La evaluación se efectúa considerando que generalmente todos los manuales sobre aprendizajes activos remarcan la importancia de la interacción promotora y de la responsabilidad social de los alumnos. En este sentido, Barkley et al. (2007) afirman que las calificaciones individuales constituyen un mecanismo para garantizar la responsabilidad de cada uno.

Los grupos de expertos son evaluados por los correspondientes profesores responsables a partir de los cuadros de evaluación o rúbricas que cada docente diseña y facilita a los estudiantes con anterioridad ( $r 1$ en Tabla 8). La evaluación individual de los alumnos depende del profesor de cada disciplina, pudiendo esta ser: prueba escrita, oral y resolución de ejercicios, entre otras. También se facilitan instrumentos para que los propios miembros del equipo se autoevalúen tanto en el logro de los objetivos como en el grado de adquisición de las competencias específicas relacionadas con su ámbito de conocimiento (r2).

En cuanto a los grupos productores, los que realizaran la maqueta, se establece un mecanismo común de evaluación de las competencias transversales independientemente de la disciplina de origen. En primer lugar, cada estudiante debe autoevaluarse y evaluar también el resto de compañeros de su grupo a partir de una rúbrica diseñada por ellos, que posteriormente es revisada y validada por el 
En segundo lugar, el guión radiofónico y la maqueta se evalúan mediante el instrumento de la rúbrica ( $\mathrm{r} 3$ y r5). El profesorado elabora una rúbrica que sirva para que tanto los docentes como los propios estudiantes evalúen las diferentes maquetas de los programas de radio.

Como muestra la Tabla 8, el plan de evaluación propuesto permite evaluar todas las competencias definidas en el proyecto, tanto las específicas como las transversales. No obstante, se hace especial hincapié en las competencias transversales comunicarse oralmente y por escrito (CT02) y trabajar en equipo (CT04) por ser estas las que están asignadas en la totalidad de las asignaturas implicadas.

Finalmente, el grupo que haya alcanzado la mejor valoración graba el programa con el apoyo técnico de una emisora profesional de radio. Esto es fundamental ya que constituye un reconocimiento y es una gran motivación. El programa piloto será difundido en la red a través de la página principal de la Universidad de Girona.

Tabla 8. Actividades, instrumentos de evaluación y competencias

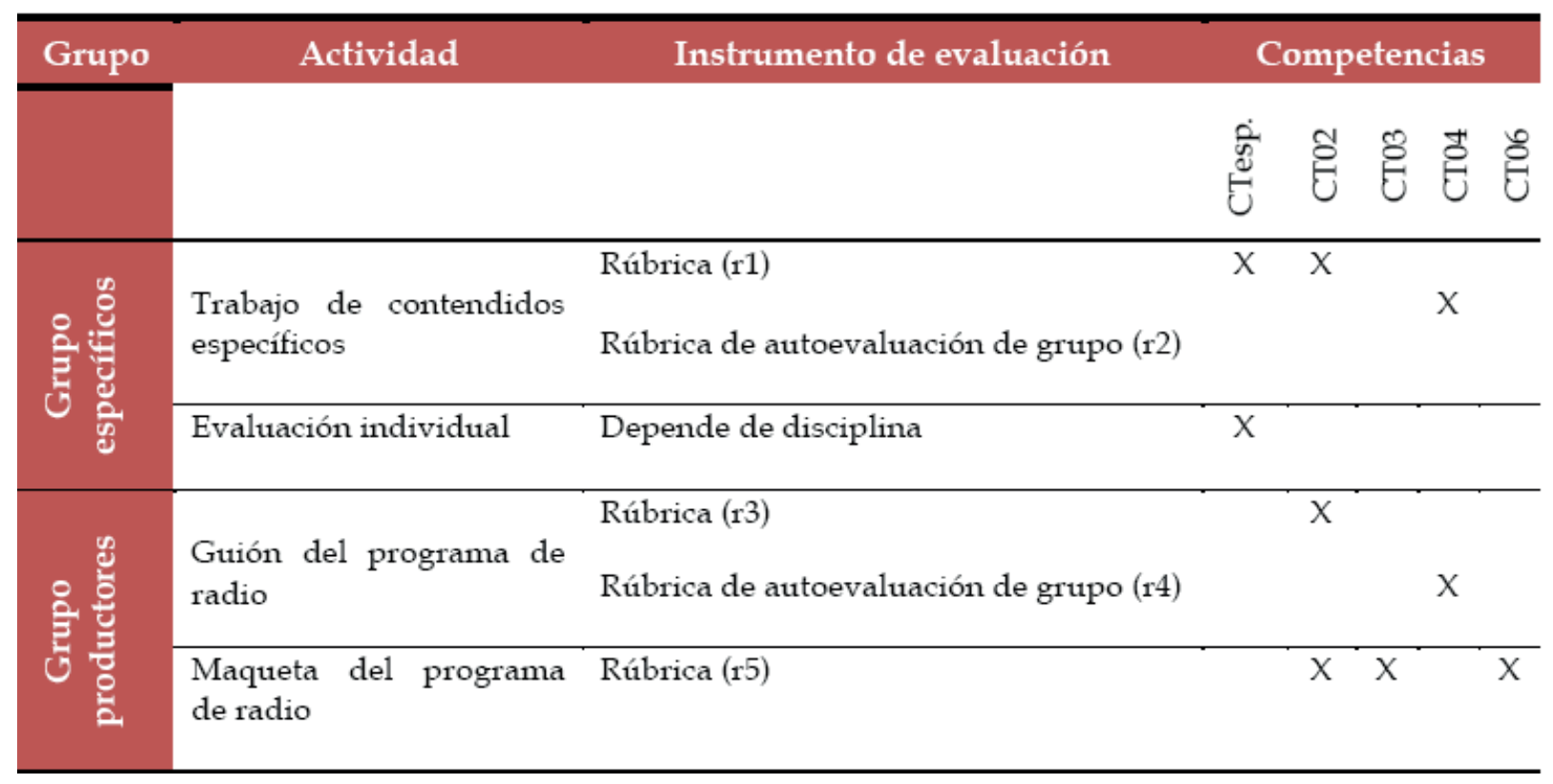

\section{CONCLUSIONES}

El proyecto Arguiñano está diseñado para trabajar las competencias propias de cada materia implicada en esta idea de trabajo, pero además, para facilitar que se puedan adquirir, de forma efectiva, competencias transversales básicas y conocimientos muy útiles en pleno siglo XXI, relacionados con aspectos de la comunicación. A modo de ejemplo, en un entorno de trabajo clásico y tradicional, los alumnos de Procesos de fahricación del grado en ingeniería mecánica de la universidad de Girona 
Del mismo modo que los estudiantes de Teoría y técnica de lenguaje radiofónico del grado en publicidad y relaciones públicas jamás aprenderían a identificar un proceso científico que les lleva a poder explicar cómo se fabrican utensilios que forman parte de nuestra vida doméstica. Arguiñano se convierte en la excusa perfecta para hacer aflorar ciencia a partir de su programa de televisión.

El aspecto más novedoso de esta fórmula reside en la combinación, en un mismo proceso de aprendizaje, de alumnos que proceden de lugares, enseñanzas y aficiones tan distintas como las que se derivan de los diferentes estudios del equipo docente involucrado, que abarca desde la publicidad y relaciones públicas hasta la ingeniería y la física. Esta mezcla de alumnado obliga a los estudiantes a tener que cambiar el lenguaje propio de su ámbito del saber por uno más llano que facilite a los otros alumnos entender "la ciencia que se cocina en la cocina".

En el proyecto planteado las competencias específicas se evalúan desde cada disciplina de origen, mientras que las competencias transversales, fijadas para el conjunto de los estudios de la Universidad de Girona, se evalúan con el trabajo realizado por los grupos con alumnos de diferentes asiganturas, intentando simular situaciones como las que se pueden encontrar en su futuro ejercicio profesional y que son precisamente las que se intentan trabajar con las competencias transversales.

Este proyecto se aplicará en dos fases. En la primera se realizará una prueba piloto a un grupo de alumnos de pequeñas dimensiones durante el segundo semestre de este curso 2011-2012. Los resultados obtenidos permitirán evaluarlo, adaptarlo y modificarlo para desplegarlo a mayor escala en una segunda fase. No obstante, la aplicación de este proyecto a gran escala, considerando que en la mayoría de especialidades implicadas en el mismo hay una media de 90 alumnos, requiere de adaptar mejor dos aspectos claves: la evaluación y la gestión de grupos grandes. Relacionado con la evaluación, es necesario profundizar en cómo hacer una evaluación de forma completamente objetiva y práctica cuando se trabaje con grupos numerosos.

Y relacionado con la gestión de grupos grandes, es necesario buscar técnicas y métodos que permitan limitar y acortar la dedicación del tiempo del profesorado docente en las tareas de guiado y de autorización de los trabajos que se desarrollan durante el proceso. En este sentido, el uso de entornos virtuales de aprendizaje, como el moddle, y el uso de otras herramientas TIC que facilitan el aprendizaje y la gestión del mismo resultan claves para garantizar el éxito del proyecto propuesto. Superar este escollo es un reto para los autores y para la viabilidad del diseño.

En este artículo se describe de forma completa y detallada un proyecto basado en APP, el cual, aunque día de hoy todavía no ha sido aplicado, se prevé que la experimentación con el grupo piloto de alumnos se lleve a término a principios del 
relatar y describir en detalle, como aquí se ha demostrado, el proceso de creación y de desarrollo de un proyecto como éste, que aunque original y complejo, no es sinónimo de imposible. La experiencia que se describe en estas páginas podría muy bien ser aplicada en cualquier otro entorno académico y por eso se considera importante compartir la idea para fomentar el uso del APP en el entorno universitario. Realzando esta dimensión más creativa, que sin duda alguna conseguirá sus objetivos pero también una mayor motivación, y por consiguiente más implicación, por parte de los alumnos.

\section{REFERENCIAS}

Albober, J., Ruiz, S. \& Valero-García, M. (2003). Evaluación de la implantación de aprendizaje basado en proyectos en la EPSC (2002-2003). Trabajo presentado al XI Congreso Universitario de Innovación Educativa en Enseñanzas Técnicas, Vilanova y la Geltrú.

Anguas, J., Díaz, L., Gallego, I., Lavado, C., Reyes, A., Rodríguez, E., Sanjeevan, K.i, Santamaría, E. \& Valero, M. (2006). Una experiencia de adaptación al EEES de dos asignaturas de programación de ordenadores. Trabajo presentado al IV Congreso Internacional de Docencia Universitaria e Innovación.

Barkley, E. F., Cross, K. Patricia, M., Claire, H. (2007). Técnicas de aprendizaje colaborativo. Madrid: Ministerio de Educación y Ciencia y Ediciones Morata.

Benito Capa, Á., Cruz Chust, A. (2007). Nuevas claves para la docencia universitaria en el Espacio Europeo de Educación Superior. Madrid: Narcea.

BUCK INSTITUTE FOR EDUCATION. (2003). Project Based Learning Handbook: A Guide to Standards-Focused Project Based Learning for Middle and High School Teachers. California: Buck Institute for Education.

Escoda I Acero, M. L., Planella Morató, J., Soler I ortega, M., Suñol I Martínez, J. J. (2009). Experiències i reflexions portades a terme en l'avaluació de competències en assignatures de l'àrea de Física Aplicada. Recuperado el 20 de agosto de 2011 de, $\underline{\text { hdl.handle.net/10256/1960. }}$.

Marina, J. A. (2010). La educación del talento. Barcelona: Ariel.

Valero, M. (2007). L'aprenentatge basat en projectes en els ensenyaments tècnics. Perspectiva escolar, 318: 42-50.

Yáñiz Álvarez, C., Villardón Gallego, L. (2006) Planificar desde competencias para promover el aprendizaje. El reto de la sociedad del conocimiento para el profesorado universitario. Bilbao: Universidad de Deusto. 


\section{AGRADECIMIENTOS}

Los autores del artículo quieren agradecer a Bainet Televisión la cesión gratuita del material audiovisual necesario para llevar a cabo el proyecto Arguiñano. Asimismo, también agradecen al Instituto de Ciencias de la Educación Josep Pallach (ICE) de la Universidad de Girona el esfuerzo por impulsar y financiar la creación de esta Red de Innovación Docente.

\section{Sílvia Espinosa Mirabet}

Periodista y doctora en comunicación audiovisual. Profesora de lenguaje radiofónico y géneros audiovisuales, ejerce su docencia en el Grado de Publicidad y en el máster de Comunicación Cultural de la Universidad de Girona. Sus investigaciones se centran en las nuevas metodologías docentes y en el análisis de formatos y géneros audiovisuales, especialmente de la radio. Forma parte del grupo de investigación ARPA de la UDG. Ha sido profesora de comunicación audiovisual y de periodismo en la Universitat autònoma de Barcelona (UAB) y en la Universitat Rovira i Virgili (URV, Tarragona) y ha trabajado como profesional en la Cadena SER, en el País o el Diari de Barcelona.

\section{Jaume Puig-Bargués}

Doctor Ingeniero Agrónomo y Profesor Titular de Ingeniería Agroforestal de la Universidad de Girona, donde imparte docencia en el Grado en Ingeniería Agroalimentaria, del que actualmente es el coordinador. Ha participado en distintos proyectos de innovación docente centrados en la implantación de ejercicios de autoevaluación en una intranet docente y en el aprendizaje basado en proyectosHa sido investigador en 5 proyectos de investigación financiados por el Gobierno de España y en otros 9 financiados por distintas empresas e instituciones. En 2004 realizó una estancia de investigación en la Kansas State University (EE.UU.).

\section{Inés Ferrer Real}

Doctora Ingeniero Industrial y profesora lectora de la Universidad de Girona, donde imparte docencia relacionada con procesos de fabricación y planificación de procesos en el Grado de Ingeniería Mecánica y en los estudios de Ingeniería Industrial. También imparte docencia en el máster oficial Business Innovation and Technology Management. Ha realizado una estancia de investigación en la Escuela Técnica Superior de Ingenieros Industriales de Madrid en 2002 y otra en la Universidad de Cambridge en 2005. 


\section{Marianna Soler i Ortega}

Doctora en Física ambiental. Profesora de Fundamentos de Física y de Instrumentos de Medida Medioambiental, ejerce su docencia en los Grados de Ingeniería Mecánica, Agroalimentaria y en el Máster en Ciencia y Tecnología del Agua de la Universidad de Girona. Su actividad investigadora se centra en el transporte de sedimentos en sistemas acuáticos y en la determinación de los patrones sinópticos en el Mediterráneo occidental. Ha realizado proyectos de colaboración con investigadores de la Universidad de Princeton especializados en modelización de sistemas acoplados océano-clima. Ha participado en 4 proyectos de ámbito nacional y pertenece al grupo reconocido por la Generalitat de Catalunya como Grupo de Calidad. 